\title{
VENEREOPHOBIA IN THE MALE*
}

\author{
BY \\ H. L. ROGERSON
}

Norwich

During recent months the incidence of venereophobia at the clinics of which I am in charge has increased. This increase has been confined almost entirely to male patients, some of whom have attended for the first time, while the others have been old patients of the clinics. This may be because the signs of venereal disease or supposed venereal disease are more obvious in the male, and not necessarily because the male worries more over the results of illicit sexual intercourse. Again, it might possibly be due to the effect of V.D. propaganda but there has not, as far as I know, been any recent local propaganda campaign. The presence of the phobia and its cure or lack of cure make no difference to the incidence of venereal disease in the country. But to the sufferer it is often more distressing than a real attack of gonorrhoea or syphilis to the average patient.

The typical case is one in which a patient, having been discharged cured from a clinic some weeks or months before, returns thinking he has a relapse. Tests are taken and he is found to be free from infection. After the appropriate interval of surveillance he is reassured and again discharged, but after a further interval, probably shorter than the last, he is back again. He may, or may not, have run a further risk of infection, and those not reexposed are the most difficult patients. Negative tests followed by reassurance do not result in cure ; the patient does not appear convinced, and one almost wishes that he might have a profuse gonococcal discharge, which by its very disappearance after treatment, would convince him.

I have recently seen a man who first attended my clinic three years ago suffering from venereophobia. He was almost beside himself with fear, and very soon required admission to a mental hospital. One of the first things he did after his discharge from the mental hospital was to contract primary syphilis. He had a typical primary chancre which was quite large enough for him to see himself.

* Received for publication June 2, 1951.
From that moment his mental symptoms have disappeared, and he has attended regularly for treatment, being obviously very pleased to be able to do so.

\section{Diagnosis}

The contrast as regards clinical and laboratory findings between the normal patient and the sceptical patient who keeps on returning to the clinic makes diagnosis easy. It is frequently so easy that the actual mode of entry of the patient into the consulting room suggests the diagnosis at once. While the diagnosis may be easy, the treatment is often difficult.

Is there a reason for the patient's disbelief when efforts are made with confidence to reassure him ? If there is a reason, can it be found? If it can be found, can the treatment be easier and more successful? The answer to all these questions is "yes", with the proviso, "not all the time", but "most of the time".

The patient has probably been reading all the popular literature on the subject of venereal disease which he can obtain. In any case he has been looking out for symptoms and signs, and he has found one or more signs which he believes to be evidence of venereal disease. What he has found may only be some normal phenomenon of which he himself was not previously aware, and no surface lesion can be too small for the keen eyes of the phobic patient.

Now, usually and unfortunately, he does not volunteer the information exactly as he himself has found it. Perhaps he thinks that if he were to disclose his own findings, they would be waved aside too easily as irrelevant. Or perhaps he is intent on satisfying himself that the venereologist knows his job and can find the sign. He states his fear of a relapse, or that he sometimes has a discharge from his penis, or he may complain of vague aching in the genital region. Usually he complains of a symptom or sign which he knows to be the recognized accompaniment of a venereal infection. He may think his own discovery of insufficient magnitude, but whatever the train of thought one 
thing is quite certain, that the venereologist must discover this sign, point it out, and explain its harmlessness to the patient. Herein lies the success of the treatment.

\section{Treatment}

Successful treatment depends on the recognition and demonstration to the patient of the sign he has found, and the explanation in terms which he can understand that this sign is not that of an active venereal infection. If the patient realizes that his suspicious sign has been considered he is more likely to be satisfied than by confident reassurance of a general nature that he is not suffering from disease. In the latter case he thinks that something has been missed, and of course it might be missed very easily since it is probably a normal phenomenon, or a lesion not connected with venereal disease.

If the signs which worry phobic patients are known, and leading questions with reference to them are put to the patient under consideration, he will readily admit the presence of the one which has been troubling him and from that moment his attitude will become less sceptical. Many patients attending the clinic with venereophobia for the first time appear surly and aggressive and this no doubt is part of their anxiety state.

During the recent "epidemic" of phobia, attempts to discover these signs were made, and the knowledge acquired was used in the interrogation of later patients. Although the complaint of the patient was quite often that of a symptom, the hidden " abnormality" was always a sign.

Nine signs were found; those numbered 1 to 4 were commonly encountered, but the remaining five were not common.

(1) Spermatorrhoea.-This was reported as a discharge and had invariably followed defaecation. Although this probably indicated recent sexual activity, and the presence of constipation, this side of the question was not explained to the patient. The explanation given was to the effect that its production resembled that which occurred when prostatic massage was resorted to during test of cure for gonorrhoea; that is, that the passage of faeces in close proximity to the gland did the work of the examining finger in the case of the test of cure. The discharge thus obtained was merely normal secretion.

(2) Sticky Meatus.-This sign was found in every case to be the result of vigorous "milking" of the penis. When asked to show his penis, the patient almost invariably carried out this operation, and in one case the amount of force used was quite astonishing. Although during treatment of an original attack of gonorrhoea the patients had been warned against "milking" the penis, some of the phobic cases had forgotten or disregarded this advice. A smear of the material was taken, and if pus cells were absent, the patient was reassured by the explanation that the discharge he had seen was the normal product from glands within the penis, increased in amount and expressed at the tip by his own squeezing.

(3) Threads in the Urine.-The phobic patient examines his urine carefully and discovers threads if he has had previous urethritis, gonococcal or non-gonococcal. It was explained that these were due to a change in the character of the lining of the penis brought about by the previous infection, and the patient usually then appeared satisfied that their presence did not indicate present infection.

(4) Phosphaturia.--This was usually reported by the patient as a discharge after micturition as opposed to a discharge of spermatorrhoea after defaecation. A sudden milkiness appearing in the stream towards the end of the act of micturition was interpreted by the patient as a discharge. If I am fortunate enough to obtain a specimen passed on the spot by the patient which contains phosphates, the explanation, followed by his watching their disappearance on the addition of acetic acid, works like a charm.

(5) Septic Spots and Warts.

(6) Ducts of Smegma Glands.

(7) Presence of Smegma.

(8) Superficial Burns caused by strong disinfectants used by the patient.

(9) Normal Sebaceous Glands and Hair Follicles of the ventral surface of the root of the penis, and sometimes of the scrotum.

As indicated above these last five signs are not common but are well worth bearing in mind.

Having questioned the patient with regard to these signs and explained any which might have been troubling him, the physician's next step is to consider how frequently and for how long the patient should attend. Of course all the tests for the exclusion of venereal disease should be carried out, even in the case of the patient who states that he has never had sexual intercourse. As stated above, the patient is often aggressive and in no mood for reassurance at his first attendance, but after a few attendances he will probably become transformed into a friendly and receptive person. I make a practice of seeing the phobic patient once a week at first. If of his own free will, he lengthens this period of time between visits, which is rare, I take this as a sign of very good progress. It is important that blood be taken at each attendance, for the patient sets great store by the blood test. The length of time of attendance varies with the progress but can be gauged by the state of the patient's outlook. I am sure that any attempt to get rid of him prematurely will result in failure of the treatment, which is, after all, a catalogue of " placebos", consisting in words and tests, with blood tests always to the fore. 